\title{
Analytical study of the swing-by maneuver in an elliptical system
}

\author{
Alessandra F.S. Ferreira ${ }^{1} \cdot$ Antônio F.B.A. Prado ${ }^{1}$. Othon C. Winter ${ }^{2}$. Denilson P.S. Santos ${ }^{3}$
}

Received: 15 August 2017 / Accepted: 22 December 2017 / Published online: 4 January 2018

๑) Springer Science+Business Media B.V., part of Springer Nature 2018

\begin{abstract}
The objective of the present paper is to derive a set of analytical equations that describe a swing-by maneuver realized in a system of primaries that are in elliptical orbits. The goal is to calculate the variations of energy, velocity and angular momentum as a function of the usual basic parameters that describe the swing-by maneuver, as done before for the case of circular orbits. In elliptical orbits the velocity of the secondary body is no longer constant, as in the circular case, but it varies with the position of the secondary body in its orbit. As a consequence, the variations of energy, velocity and angular momentum become functions of the magnitude and the angle between the velocity vector of the secondary body and the line connecting the primaries. The "patched-conics" approach is used to obtain these equations. The configurations that result in maximum gains and losses of energy for the spacecraft are shown next, and a comparison between the results obtained using the analytical equations and numerical simulations are made to validate the method developed here.
\end{abstract}

$\square$ A.F.S. Ferreira

aleferrazsilva@hotmail.com

A.F.B.A. Prado

antonio.prado@inpe.br

O.C. Winter

ocwinter@gmail.com

D.P.S. Santos

denilson.paulo@gmail.com

1 Instituto Nacional de Pesquisas Espaciais - INPE, São José dos Campos, SP, Brazil

2 Grupo de Dinâmica Orbital e Planetologia, Univ. Estadual Paulista - UNESP, Guaratinguetá, SP, Brazil

3 Univ. Estadual Paulista - UNESP, São João da Boa Vista, SP, Brazil
Keywords Astrodynamics · Swing-by maneuvers · Spacecraft trajectories . Elliptical systems

\section{Introduction}

The gravity assist, or swing-by, is a maneuver where the spacecraft makes a rotation around the secondary body of a system composed by two celestial bodies, with the purpose of using the relative motion of this body with respect to the main body to modify its trajectory (velocity, energy and angular momentum). The maneuver can either increase or decrease the velocity of the spacecraft and, consequently, its energy and the whole trajectory. The gain or loss of energy can be favorable to the mission, if the goal is an escape or capture of the spacecraft by the body (Havnes 1969; Horedt 1972, 1974, 1976; Cline 1979; Nock and Upholf 1979; Bao et al. 2015). The main focus is the fuel economy and, consequently, the reduction in the cost of the mission.

Several works related to swing-by trajectories were developed and are available in the literature. One of the first was made by Minovitch (1961), followed by Clarke (1962), Niehoff (1966), Hollister and Prussing (1966), Deerwester (1966), Diehl and Myers (1987), and Qi and Xu (2015), among others. In terms of practical applications, considering missions that used this type of maneuver, Flandro (1966) designed the Voyager missions, which were later sent to the outer planets. The gravity assist was also applied to the mission Galileo, which was sent to Jupiter (D'Amario et al. 1981, 1982; Byrnes and D'Amario 1982), Mariner 10 and the Messenger missions sent to Mercury (Dunne and Burgess 1978; McNutt et al. 2004, 2006; Grard 2006); and LCROSS, which was launched in 2009 and, after five days from launching, made a swing-by around the Moon with 
the goal of entering a polar orbit around the Moon (NASA 2009).

Broucke (1988) made an analytical study of the gravity assist maneuver, presenting the increment of velocity, energy and angular momentum, for a system where the primaries are in circular orbits and in planar motion. The results are written as functions of the three basic parameters that determine the swing-by maneuver, which are the angle and velocity of approach and the periapsis distance (Broucke 1988). This paper also examined in detail the parameters for the maximum gain and the maximum loss of energy to cover different aspects of the problem. It is also performed numerical explorations using the restricted threebody problem to classify the types of orbits resulting from this maneuver. After that, Prado (1996) made an extension of this work to include the application of an impulse at the spacecraft during the close encounter to get more effects from the maneuver. In a sequence, Casalino et al. (1999a) expanded this analytical study to cover the situation where the impulse is applied in different points of the trajectory. There are several other works considering the study of the swing-by maneuver combined with an impulse in the spacecraft based on numerical integration (Prado 1997; Casalino et al. 1999b; Santos et al. 2008; Silva et al. 2013; Ferreira et al. 2015, 2017a, 2017b, 2017c; Piñeros and Prado 2017).

Then, the objective of the present paper is to make an analytical study of the pure gravity swing-by maneuver (without impulse), presenting the equations for the velocity, energy and angular momentum variation as functions of the basic parameters of the swing-by, for systems where the primary bodies are in elliptical orbits. Prado (1997) and Ferreira et al. (2017a) performed numerical studies for systems in elliptical orbits, using the elliptic restricted three body problem, but in both cases considering also an impulsive maneuver. Those studies showed interesting aspects of the problem, indicating the necessity of making analytical studies for the elliptical case, similar to those available for the circular problem.

Next, Sect. 2 presents the definition of the problem. In Sect. 3, the configurations of maximum gains and losses of energy are shown. In Sect. 4, a comparison is made between the results obtained by the analytical and numerical method and, finally, the conclusions of the work are shown.

\section{Formulation of the problem}

This study considers a system with primary bodies in elliptical orbits around a common center of mass. The spacecraft $\left(M_{3}\right)$ makes the maneuver around the secondary body $\left(M_{2}\right)$ and uses the gravity of this body to modify its trajectory. The variations of velocity, energy and angular momentum

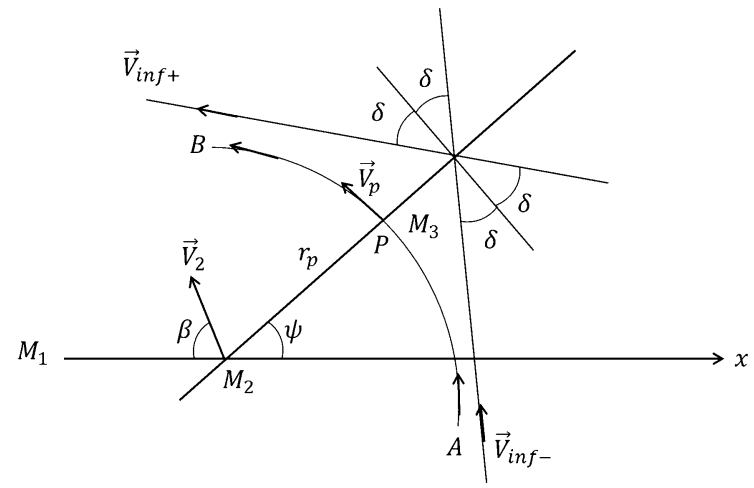

Fig. 1 Geometry of the swing-by maneuver in an elliptical system

are calculated with respect to the primary body (the one with a larger mass) of the system $\left(M_{1}\right)$.

The derivations of the equations follow the usual approximation given by the "patched-conics" model, as done in the circular case (Broucke 1988). This is a method to simplify the calculations of the trajectory of a spacecraft in a multibody environment. When the spacecraft is within the sphere of influence of a body (Bate et al. 1971), the gravitational force of the other body is neglected. The trajectory is then analyzed by splitting the total motion of the spacecraft in three parts dominated by the "two-body" dynamics. The first part is when the spacecraft is within the sphere of influence of the largest central body and the gravitational force of the smaller body is disregarded, so the system considered is the "spacecraft-largest body". From this step it is possible to find the geometry and the velocity of approach of the spacecraft with respect to the smaller body of the system. Then comes the second part of the motion, and the study considers now the "two-body" system "spacecraft-secondary body". It gives the geometry and velocity for the spacecraft when leaving the secondary body. Then, the third and last part of the motion considers the "two-body" "spacecraft-largest body" again, after the maneuver. After those three steps the new orbit of the spacecraft is obtained, based on a series of three "two-body" problems.

The numerical study integrates the equations of motion of the Elliptic Restricted Three-Body Problem (Szebehely 1967), obtaining the trajectories always considering the presence of all the bodies involved in the system.

An important effect of the eccentricity of the primaries, as far as the swing-by maneuver is considered, is that this eccentricity and the true anomaly of the secondary body at the time of the maneuver change the behavior of the velocity of the secondary body with respect to the first primary $\left(\vec{V}_{2}\right)$, which is no longer constant. This is a very important parameter in the effects generated by this maneuver. Figure 1 shows the geometry of the maneuver for the elliptical case, adapted from the circular maneuver (Broucke 1988).

In this figure, $P$ is the periapsis of the orbit of the spacecraft around the secondary body. The periapsis radius of the 


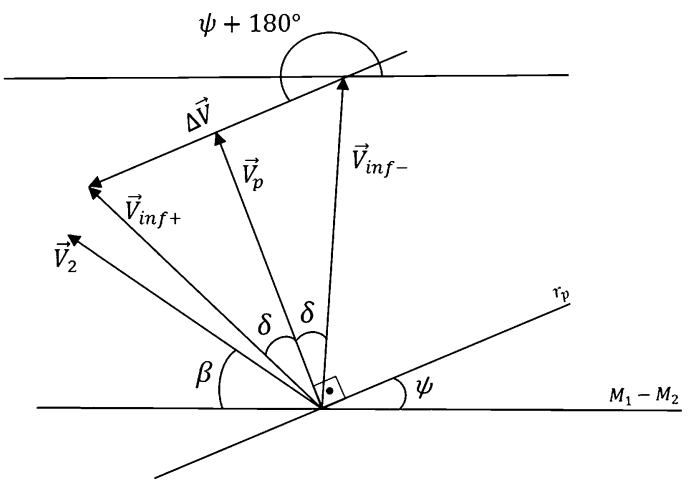

Fig. 2 Velocity vectors involved in the swing-by maneuver for an elliptical system

orbit of the spacecraft around the secondary body is $r_{p}$, and $\vec{V}_{p}$ is the velocity at this point. $\psi$ is the angle of approach, which defines the orientation of the swing-by maneuver. The velocity of the secondary body around the center of mass of the system is $\vec{V}_{2}$, which, in the case of elliptical orbits, varies according to the position of the body in its orbit. $\left(180^{\circ}-\beta\right)$ is the angle between $\vec{V}_{2}$ and the line connecting the primaries. $\vec{V}_{\text {inf }- \text { and }} \vec{V}_{\text {inf }}$ are the velocities of approach and departure with respect to $M_{2}$, respectively. These velocities are equal in magnitude, so $V_{\text {inf- }}=V_{\text {inf }+}=V_{\text {inf }}$, but not in terms of directions. Their components are calculated from Fig. 2. The total deflection of the maneuver is denoted by $2 \delta$, where $\sin \delta=\frac{1}{\left(1+\frac{r_{p} V_{\text {inf }}^{2}}{\mu}\right)}$, with $\mu$ being the gravitational parameter of the secondary body.

Equations (1) and (2) present the components of the velocities $\vec{V}_{\text {inf- }}$ and $\vec{V}_{\text {inf }+}$ :

$\vec{V}_{\text {inf- }}:\left\{\begin{array}{l}\dot{X}=V_{\text {inf }} \cos \left(90^{\circ}+\psi-\delta\right)=-V_{\text {inf }} \sin (\psi-\delta), \\ \dot{Y}=V_{\text {inf }} \sin \left(90^{\circ}+\psi-\delta\right)=+V_{\text {inf }} \cos (\psi-\delta),\end{array}\right.$

$\vec{V}_{\mathrm{inf}+}:\left\{\begin{array}{l}\dot{X}_{+}=V_{\mathrm{inf}} \cos \left(90^{\circ}+\psi+\delta\right)=-V_{\mathrm{inf}} \sin (\psi+\delta), \\ \dot{Y}_{+}=V_{\mathrm{inf}} \sin \left(90^{\circ}+\psi+\delta\right)=+V_{\mathrm{inf}} \cos (\psi+\delta) .\end{array}\right.$

The velocity of the spacecraft relative to $M_{1}$, before the close encounter, is given by $\vec{V}_{i}=\vec{V}_{\text {inf- }}+\vec{V}_{2}$ and, after the close encounter, it is $\vec{V}_{o}=\vec{V}_{\text {inf+ }}+\vec{V}_{2}$. The variation of the velocity of the spacecraft is given by $\Delta \vec{V}=\vec{V}_{o}-\vec{V}_{i}$, with components $\Delta \dot{X}$ and $\Delta \dot{Y}$ given by Eq. (3):

$\left\{\begin{array}{l}\Delta \dot{X}=-2 V_{\text {inf }} \sin \delta \cos \psi \\ \Delta \dot{Y}=-2 V_{\text {inf }} \sin \delta \sin \psi\end{array}\right.$

Then, the variation of the velocity as a function of the fundamental parameters of the swing-by is

$\Delta V=|\Delta \vec{V}|=2 V_{\text {inf }} \sin \delta$.
Note that the effect of the secondary body gravity basically consists in rotating the velocity vector by an angle $2 \delta$. The next step is to calculate the energy variation, which is given by $\Delta E=\frac{1}{2}\left(\vec{V}_{o}^{2}-\vec{V}_{i}^{2}\right)$, or $\Delta E=\vec{V}_{2} \cdot \Delta \vec{V}$ (Broucke 1988; Barger and Olsson 1973). Decomposing the orbital velocity of the secondary body in the plane, the result is $\vec{V}_{2}=\left(-V_{2} \cos \beta, V_{2} \sin \beta\right)$, where $\beta=\cos ^{-1}\left(-\frac{V_{r}}{V_{2}}\right)$. $V_{r}=e \sqrt{\frac{(1-\mu)}{a\left(1-e^{2}\right)}} \sin \nu$ is the radial velocity of the secondary body and $V_{2}=\sqrt{(1-\mu)\left(\frac{2}{d}-\frac{1}{a}\right)}$ the respective magnitude. $a$ is the semi-major axis of the orbit of the primary, $e$ the eccentricity, $v$ the true anomaly of $M_{2}$, and $d=\frac{a\left(1-e^{2}\right)}{1+e \cos v}$ the distance between the primary bodies.

Therefore, the variation of energy of the spacecraft, as a function of the three independent parameters related to the swing-by and the parameters related to the eccentric orbit of the secondary body around the primary, is given by $\Delta E=2 V_{\text {inf }} V_{2} \sin \delta \cos (\psi+\beta)$. So, combining the above equations, it is possible to get a complete closed formula for the variation of energy, which is shown in Eq. (5), as a function of the parameters related to the swing-by maneuver and the orbit of the secondary body around the primary:

$$
\Delta E=2 V_{\text {inf }} \sqrt{(1-\mu)\left(\frac{2}{\frac{a\left(1-e^{2}\right)}{1+e \cos v}}-\frac{1}{a}\right)} \sin \delta \cos (\psi+\beta) .
$$

In the elliptical case, $\beta=90^{\circ}$ when the secondary body is in the apoapsis or periapsis of its orbit around the main body. In these cases the energy variation is reduced to $\Delta E=$ $-2 V_{\text {inf }} \sqrt{(1-\mu)\left(\frac{2}{\frac{a\left(1-e^{2}\right)}{1+e \cos v}}-\frac{1}{a}\right)} \sin \delta \sin \psi$, which is similar to the circular case (Broucke 1988), except for the difference when computing the velocity $V_{2}$, which is different in both situations.

Figure 3 shows the energy variation (in canonical units) as a function of eccentricity and true anomaly of the secondary body for a generalized Earth-Moon system. This is a system where the ratio of the masses and the radius of the two bodies involved in canonical units, so the distance between the centers of the primaries is used as a unit, are the same of the Earth-Moon system, but the eccentricity is varied to study the effects of this parameter in the swingby maneuver. The parameter values of $r_{p}=1.1$ for radius of the Moon, $V_{\text {inf }}=1.0$ canonical unit (c.u.) and $\psi+\beta=360^{\circ}$ are used. One canonical unit of velocity is defined as the velocity of the secondary body around the primary body.

Note that in regions where the true anomaly $(v)$ is less than $90^{\circ}$ or greater than $270^{\circ}$, the energy variations are increasing functions of the eccentricity and, in the regions where true anomaly $(v)$ is between $90^{\circ}$ and $270^{\circ}$, decreasing functions of the eccentricity. The maximum energy variation 


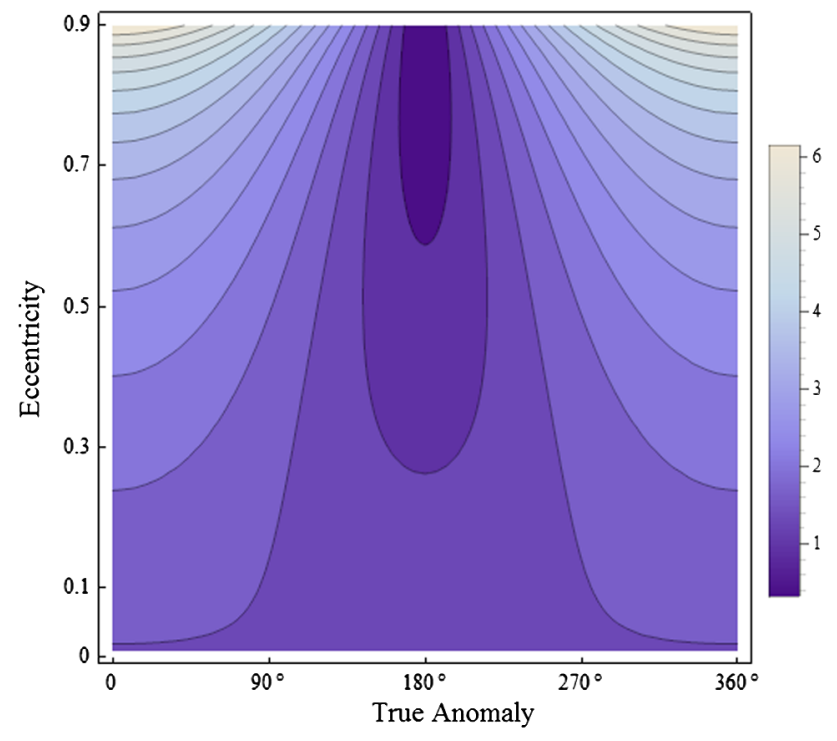

Fig. 3 Energy variation (c.u.) in the generalized eccentric Earth-Moon system

is approximately 6.0 c.u. and it occurs for the extreme values of the eccentricity and true anomaly. The minimum energy variation is in the region where the eccentricity is greater than approximately 0.6 and for $v$ close to $180^{\circ}$.

Angular momentum is a quantity associated with the spacecraft rotation. By definition, the angular momentum is equal to the cross-product between the position and the velocity vectors, so $\vec{C}=\vec{d} \times \vec{V}$. It is also known that, under the "patched-conics" model, the swing-by changes the orbit of the spacecraft instantly, so $\vec{d}$, which is the position of the secondary body with respect to the primary one at the moment of the maneuver, is constant. Then, if $\vec{C}_{-}=\vec{d} \times \vec{V}_{i}$ is the angular momentum calculated before the swing-by and $\vec{C}_{+}=\vec{d} \times \vec{V}_{o}$ is the angular momentum calculated after the swing-by, the difference between them gives the angular momentum variation, which is given by $\Delta \vec{C}=\vec{d} \times\left(\vec{V}_{o}-\vec{V}_{i}\right)=\vec{d} \times \Delta \vec{V}$. The magnitudes of $\vec{d}$ and $\Delta \vec{V}$, as well as the angle between them (see Fig. 2), are known so the calculations are easy:

$$
\begin{aligned}
\Delta C & =|\Delta \vec{C}|=2 d V_{\mathrm{inf}} \sin \delta \sin \left(180^{\circ}+\psi\right) \\
& =-2 d V_{\mathrm{inf}} \sin \delta \sin \psi \\
& =-2 \frac{a\left(1-e^{2}\right)}{(1+e \cos v)} V_{\mathrm{inf}} \sin \delta \sin \psi .
\end{aligned}
$$

Observe that, in this case, the final equation is dependent on the parameters related to the eccentricity, but not on the angle $\beta$, which characterizes the velocity of the secondary body. This angle disappears during the development of the equation, when the velocity variation is obtained.

Figure 4 shows the behavior of the angular momentum variation as a function of the eccentricity and the true

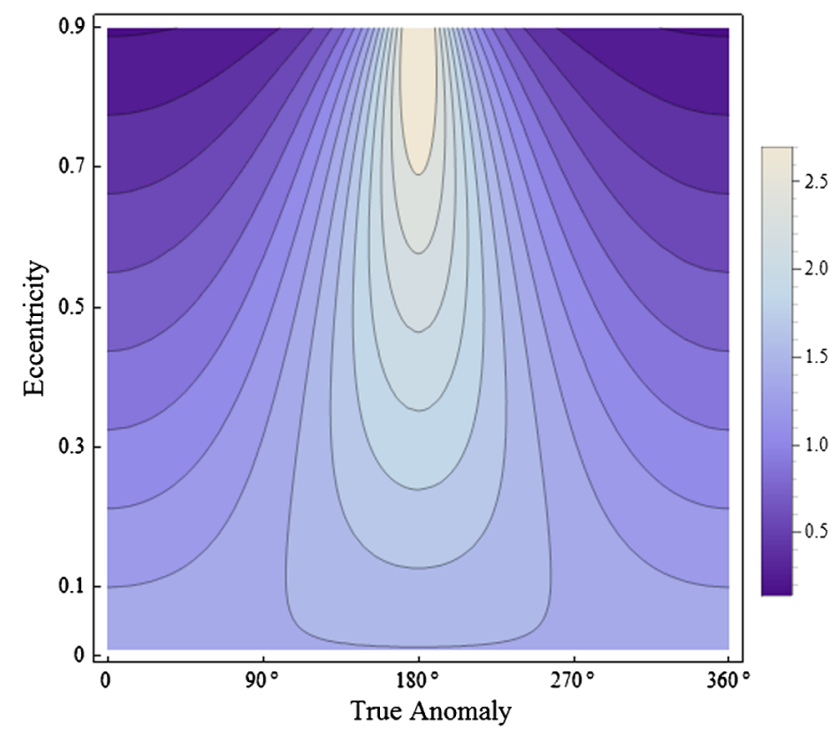

Fig. 4 Angular momentum variation (c.u.) in the generalized eccentric Earth-Moon system

anomaly of the secondary body, for the generalized EarthMoon system explained before, using $r_{p}=1.1$ for radius of the secondary body, $V_{\text {inf }}=1.0$ c.u. and $\psi=270^{\circ}$.

The angular momentum has a behavior opposite to the energy, increasing around $90^{\circ}<v<270^{\circ}$ and decreasing when $v<90^{\circ}$ and $v>270^{\circ}$, according to the increase in the eccentricity. For this case, the always positive variation shows that the spacecraft increased the angular momentum in the second orbit, after the maneuver, when compared to the first orbit.

\section{Configurations of maximum gains and losses of energy}

For systems in circular orbits, the spacecraft loses energy when $0^{\circ}<\psi<180^{\circ}$, with maximum loss when $\psi=90^{\circ}$; and it gains energy when $180^{\circ}<\psi<360^{\circ}$, with maximum gain when $\psi=270^{\circ}$ (Broucke 1988).

For systems in elliptic orbits, by examining Eq. (5), one can see that $V_{\text {inf }}, V_{2}$ and $\sin \delta$ are always positive, since $0^{\circ}<\delta<90^{\circ}$. It means that it is the orientation of the swingby with respect to $M_{2}$, given by $\psi$, and the orientation of $\vec{V}_{2}$, given by $\beta$, that defines the signal of the variation of energy. The negative values, so maneuvers with energy loss, occur when $90^{\circ}<\psi+\beta<270^{\circ}$, with maximum loss at $\psi+\beta=$ $180^{\circ}$. The region of gain is $-90^{\circ}<\psi+\beta<90^{\circ}$, with maximum gain when $\psi+\beta=0^{\circ}$ or $\psi+\beta=360^{\circ}$. It can be seen that the effect of the swing-by is zero when $\psi+\beta=90^{\circ}$ or $\beta+\psi=270^{\circ}$. This information is clearly shown in Fig. 5, which shows $\Delta E$ for a system with $2 V_{\text {inf }} V_{2} \sin \delta=1$.

A map of the energy variation as a function of $\psi$ and $\beta$, for a system with the same parameters used in Fig. 5, is pre- 


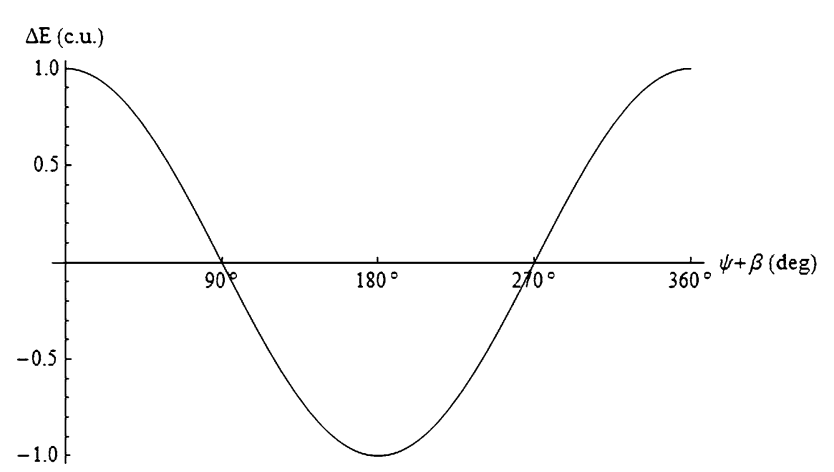

Fig. 5 Energy variation (Eq. (5)) as a function of $\psi+\beta$

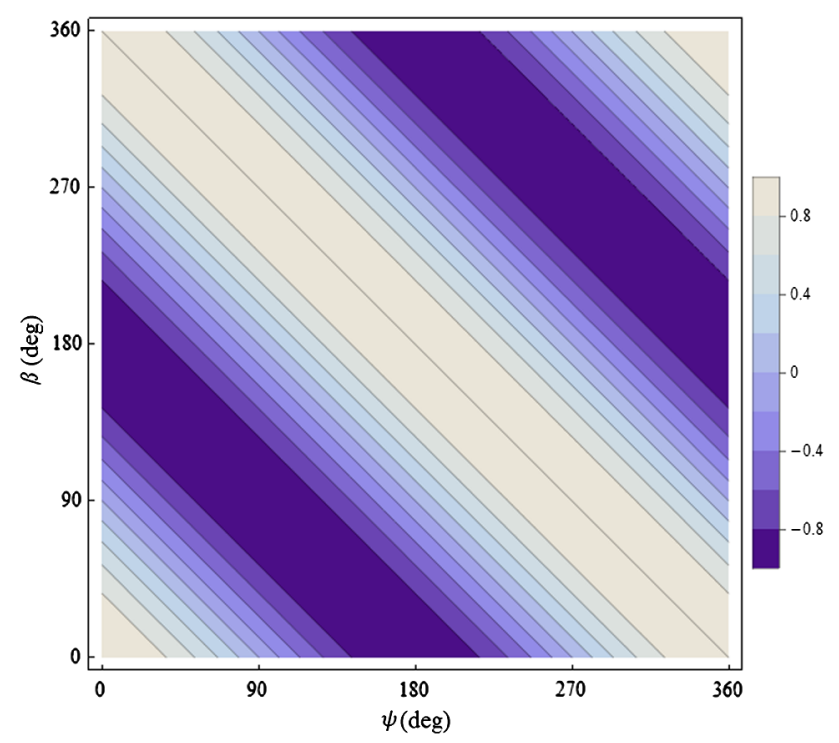

Fig. 6 Energy variation (Eq. (5)) for a system with $2 V_{\text {inf }} V_{2} \sin \delta=1$, as a function of $\beta$ and $\psi$

sented in Fig. 6. It is possible to see the effects of $\psi$ and $\beta$ in the energy variation.

If the analysis is reduced to the cases where $\psi=90^{\circ}$ and $\psi=270^{\circ}$, the energy variation, as a function of $\beta$, has the behavior shown in Fig. 7.

In the situation where $\psi=90^{\circ}$, the maximum loss of energy occurs for $\beta=90^{\circ}$ and the maximum gain for $\beta=$ $270^{\circ}$. In the case where $\psi=270^{\circ}$, the maximum loss is for $\beta=270^{\circ}$ and the maximum gain for $\beta=90^{\circ}$. The energy variation is zero when $\beta=0^{\circ}$ or $\beta=180^{\circ}$.

Note that the analysis of the maneuver for an elliptical system coincides with the circular case when the secondary body is at the periapsis or apoapsis of its orbit. This is due to the fact that, in these cases, $\beta=90^{\circ}$ and, in the circular case, $\beta$ is always $90^{\circ}$. Therefore, the point of maximum gain of energy occurs when $\psi=270^{\circ}$ and the maximum loss when $\psi=90^{\circ}$, for both cases. The swing-by has zero energy variation for $\psi=0^{\circ}$ or $\psi=180^{\circ}$.

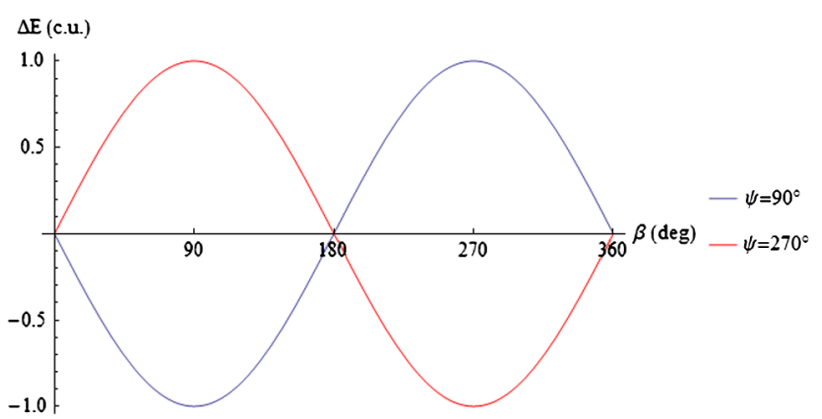

Fig. 7 Energy variation for a system with $2 V_{\text {inf }} V_{2} \sin \delta=1, \psi=90^{\circ}$ and $\psi=270^{\circ}$

\section{Comparison between the results obtained by the analytical and the numerical method}

The goal of this comparison is to validate the equations developed in the present paper, as well as to show the differences in the results for the energy variation when the study is done analytically, using the "patched conics" approximation and numerical integrating the equations of motion of the spacecraft (Eqs. (7) and (8)) given by the elliptical restricted three-body problem. In this way, it is possible to identify the errors introduced by the approximations involved in the analytical developments made here. The focus is not a detailed study of those errors, but just a quick validation of the analytical equation derived here.

$\ddot{x}=\frac{-(1-\mu)\left(x-x_{1}\right)}{r_{1}^{3}}-\frac{\mu\left(x-x_{2}\right)}{r_{2}^{3}}$,

$\ddot{y}=\frac{-(1-\mu)\left(y-y_{1}\right)}{r_{1}^{3}}-\frac{\mu\left(y-y_{2}\right)}{r_{2}^{3}}$.

In the above equations, $r_{1}$ is the distance $M_{1}-M_{3}$; $r_{2}$ the distance $M_{2}-M_{3} ; x_{1}=-\mu d \cos v, y_{1}=-\mu d \sin v$, $x_{2}=(1-\mu) d \cos v$ and $y_{2}=(1-\mu) d \sin v$ are the positions of $M_{1}$ (the primary body) and $M_{2}$ (the secondary body), respectively, in the fixed reference system originated in the center of mass of the system. $M_{3}$ is the spacecraft, assumed to have a negligible mass.

Regarding comparisons measuring the effects of the analytical approximations in the results for a swing-by maneuver, there are some references in the literature. Prado (2007) compares the analytical and numerical results of the swingby maneuver in the Sun-Jupiter system, considering the orbit of the primaries as circular. In this study it is shown that, for different initial conditions, the error between the two methods reaches up to $\pm 5 \%$. In the cases where the error is positive, the "patched-conics" model overestimates the effects of the swing-by. When the error is negative, the "patched-conics" model underestimates the effects of the maneuver. This occurred for conditions with energy changes 


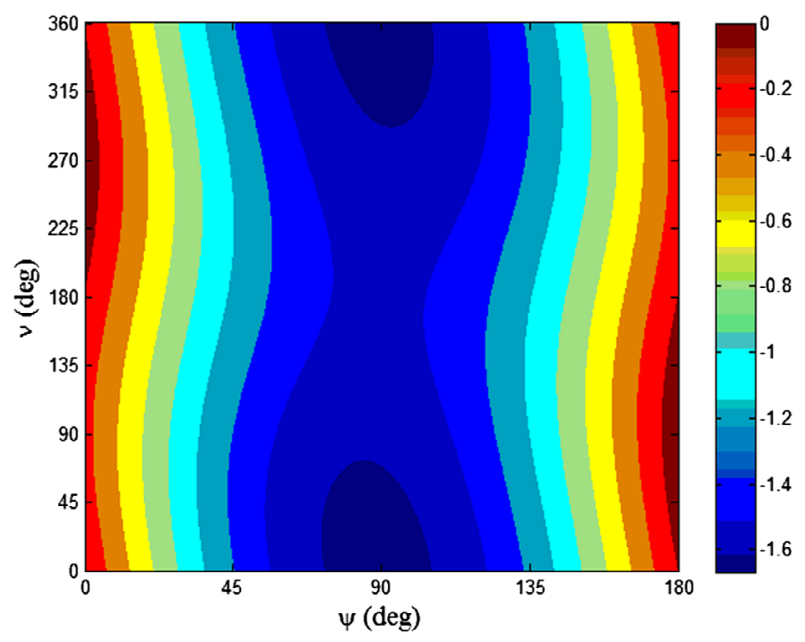

(a) $\Delta E$ numerical

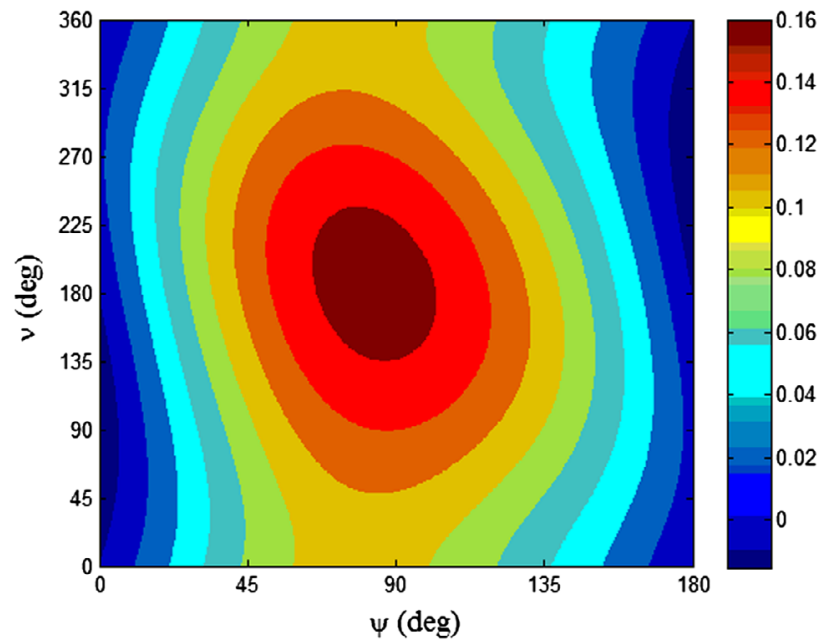

(b) Absolute Error

Fig. 8 Energy variations using the numerical method (a) and the absolute error between the methods (b), for $e=0.1$

around zero. For these situations the "patched-conics" approximation method has a limited accuracy. A more detailed study of this point is available in Negri et al. (2017).

In the present paper, the absolute error between the two models is presented by the difference between the energy variation using the patched conics method and the energy variation using the Elliptical Restricted Three-Body Problem for the generalized Earth-Moon system. Figure 8 shows the energy variations using the numerical method and the absolute error between numerical and analytical methods, for $e=0.1$, as a function of the approach angle $(\psi)$ and the true anomaly of $M_{2}(v)$.

The behaviors of the solutions for the analytical and numerical methods are similar, with differences presented in the absolute error in the cases analyzed of the same order of the circular case. This fact indicates that the analytical equations are correct and give an approximation for the maneuver as good as those used for the circular case. A detailed

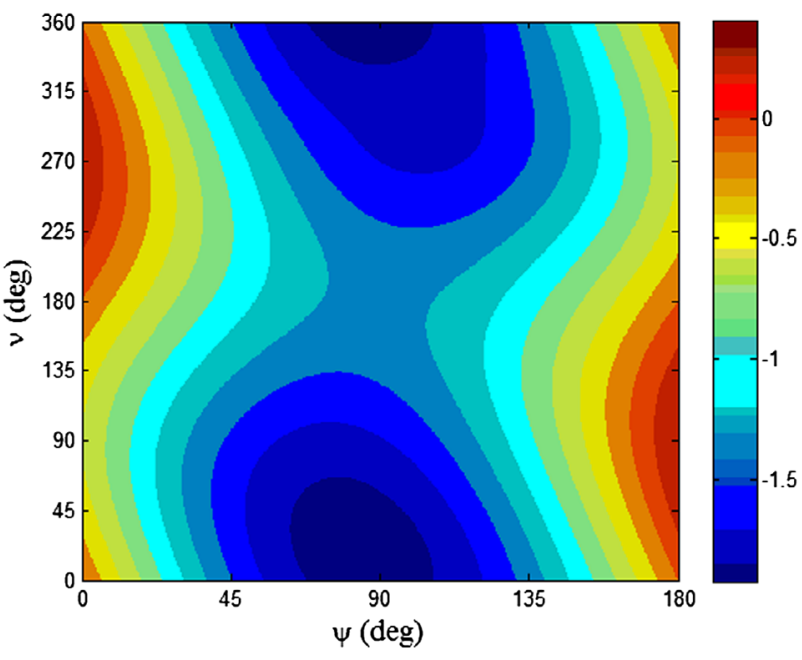

(a) $\Delta E$ numerical

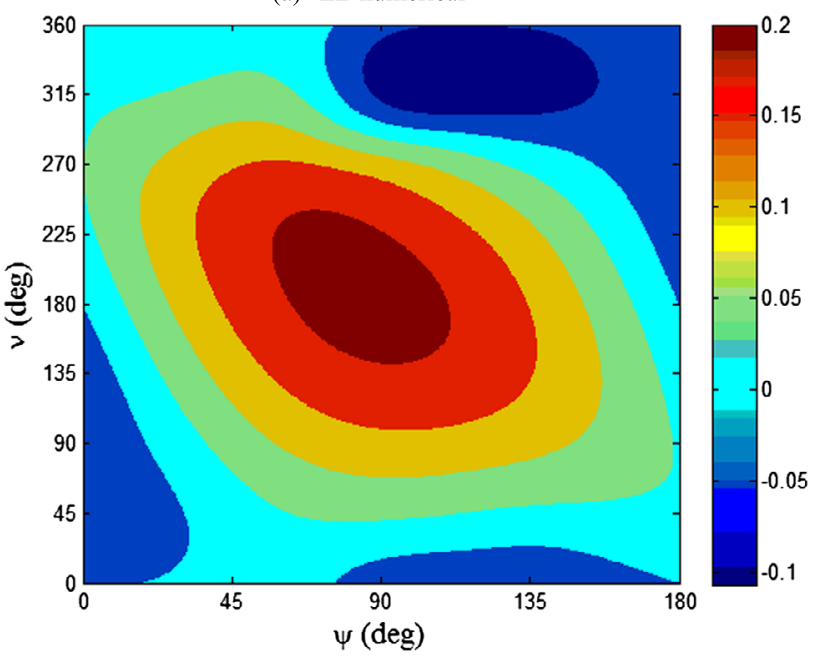

(b) Absolute Error

Fig. 9 Energy variations using the numerical method (a) and the absolute error between the methods (b), for $e=0.3$

study of this point is recently made for the circular case (Negri et al. 2017). A similar analysis is not in the scope of the present paper, but should be done to clarify this point. It is also clear that the largest differences occur when the value of $\psi$ is around $90^{\circ}$, and this difference reaches the order of 0.16 c.u. in the case simulated here. The solutions for $180^{\circ}<\psi<360^{\circ}$ are symmetrical, just with an inversion in the sign of the variations of energy, which is positive.

Figure 9 shows the energy variations using the numerical method and the absolute error, for $e=0.3$, as a function of the approach angle $(\psi)$ and the true anomaly of $M_{2}(v)$.

The first observation that can be made in Fig. 9 is the deformation of the curves of the energy variation as a function of $\psi$ and $v$, when compared with Fig. 8. Figure 10, made for $e=0.5$, shows that the higher the eccentricity, the greater these deformations. The absolute error has a small increase, and it reaches 0.2 c.u. A significant region with errors around zero is notorious. The solutions for $180^{\circ}<$ 


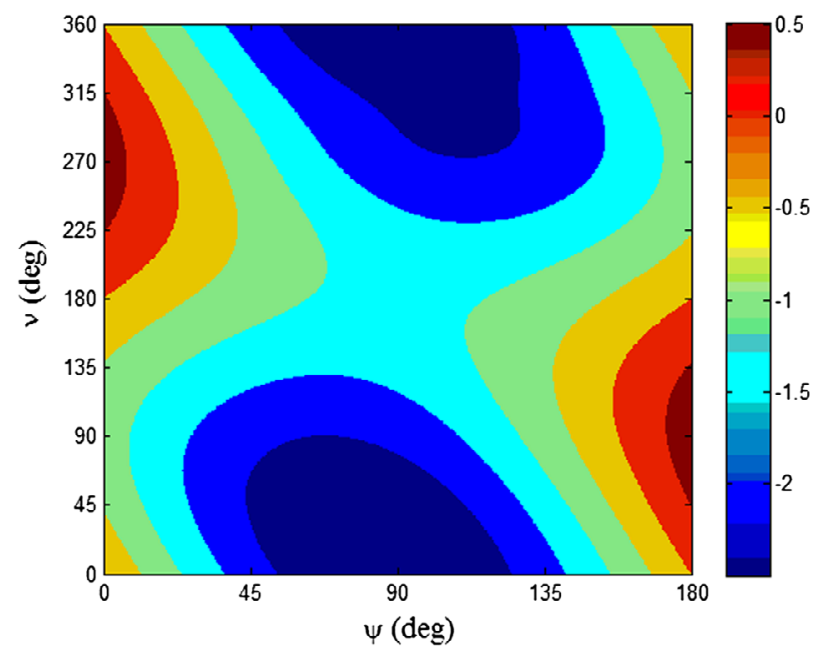

(a) $\Delta E$ numerical

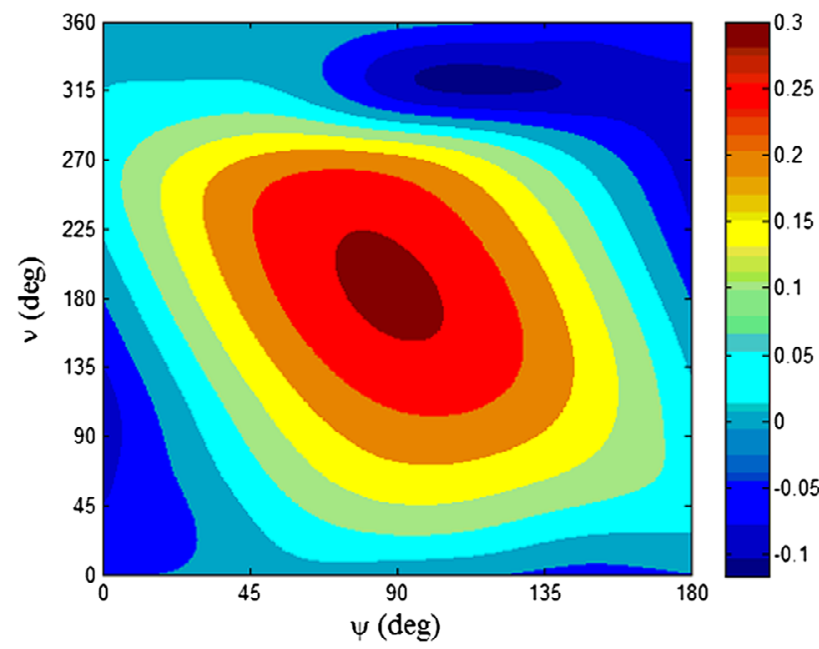

(b) Absolute Error

Fig. 10 Energy variations using the numerical method (a) and the absolute error between the methods (b), for $e=0.5$

$\psi<360^{\circ}$ are symmetrical, just with the inversion of sign explained before.

Figure 10 shows the energy variation using the numerical method and the absolute error between numerical and analytical methods for the case where $e=0.5$.

It is noted that the absolute errors reach values up to 0.3 c.u. The region with the highest errors is around $\psi=$ $90^{\circ}$. As with other eccentricities, when $\psi$ is between $180^{\circ}$ and $360^{\circ}$, with gains of energy due to the gravity, the solutions are symmetrical to the situations where $\psi$ is between $0^{\circ}$ and $180^{\circ}$, just with the inversion of sign.

The magnitude of the maximum error for $e=0.1$ (Fig. 8b) is approximately $11 \%$, for $e=0.3$ (Fig. 9b) approximately $17 \%$, and for $e=0.5$ (Fig. 10b) about 27\%, being larger than $5 \%$, the maximum result obtained for the circular case in the Sun-Jupiter system (Prado 2007). For the lowest values of eccentricity, they are similar to the values obtained in the circular case considering the Earth-Moon system (Prado 1996). This behavior is expected, since the same parameters of the Earth-Moon system were used, except for the eccentricity, in the present paper. A larger gravitational parameter for the system decreases the accuracy of the analytical model, as detailed in Negri et al. (2017). The results also show in details that the eccentricity of the primaries makes the velocity of the secondary body around the primary $\left(\vec{V}_{2}\right)$ to have values which are dependent on the position of $M_{2}$ in its orbit. For most of the cases with $\psi=270^{\circ}$ the error is negative, which means that the "patched-conics" model underestimates the energy gain near the point of the maximum effect of the swing-by. For the cases with $\psi=90^{\circ}$, the patched-conics model also underestimates the loss of energy of the spacecraft.

\section{Conclusions}

An analytical study of the swing-by maneuver for a system with elliptical orbits for the primaries was presented. Equations were derived to calculate the variation of velocity, energy and angular momentum for the swing-by maneuver as a function of the parameters that define the geometry of the maneuver and the parameters that characterize the elliptical orbit of the primary bodies.

The swing-by is developed around the secondary body of the system, and the quantities are obtained with respect to the main body of the system. The main effect of the eccentricity is the variation in the velocity of the secondary body with respect to the center of mass of the system. This velocity depends on the position of the secondary body in its orbit, so it is no longer constant as in the circular case.

Solutions for the energy variation and angular momentum for a system with parameters similar to Earth-Moon, except for the eccentricity, are presented as functions of the eccentricity of the orbit and the true anomaly of the secondary body. The configurations resulting in maximum gain and maximum loss of energy for the spacecraft are also presented and compared with the cases with circular orbits. Comparisons were made between the analytical method, obtained from the patched conics approximation, and the numerical method implemented using the elliptic restricted three-body problem.

The work shows that the position of maximum energy gain, for eccentric systems, is $\psi+\beta=0^{\circ}$ or $\psi+\beta=360^{\circ}$ and the position of maximum energy loss is $\psi+\beta=180^{\circ}$. If the secondary body is at the periapsis or apoapsis of its orbit, the position of maximum energy gain reduces to $\psi=270^{\circ}$ and the maximum loss to $\psi=90^{\circ}$. The magnitude of the absolute error, obtained in the comparison of the analyzed methods, in several cases is greater than 5\%. The magnitude of this error tends to increase when the eccentricity increases, reaching the order of approximately $0.16 \mathrm{c}$.u. for ec- 
centricity of $0.1 ; 0.2$ c.u. for eccentricity of 0.3 , and 0.3 c.u. for eccentricity 0.5 .

Acknowledgements The authors wish to express their appreciation for the support provided by grants \#406841/2016-0, 301338/2016-7 and 312813/2013-9 from the National Council for Scientific and Technological Development (CNPq); grants \#2016/14665-2, 2016/24561-0 and 2017/04643-4 from São Paulo Research Foundation (FAPESP) and the financial support from the National Council for the Improvement of Higher Education (CAPES).

\section{References}

Bao, C., Yang, H., Barsbold, B., Bayoin, H.: Capturing near-Earth asteroids into bounded Earth orbits using gravity assist. Astrophys. Space Sci. 360(2), 61 (2015)

Barger, V., Olsson, M.: Classical Mechanics: A Modern Perspective. McGraw-Hill, New York (1973)

Bate, R.R., Mueller, D.D., White, J.E.: Fundamentals of Astrodynamics, pp. 333-334. Dover Publications, New York (1971)

Broucke, R.A.: The celestial mechanics of gravity assist. In: AIAA/AAS Astrodynamics Conference, vol. 88 (1988). https:// doi.org/10.2514/6.1988-4220

Byrnes, D.V., D'Amario, L.A.: A combined Halley flyby Galileo mission. In: AIAA/AAS Astrodynamics Conference, vol. 82 (1982). https://doi.org/10.2514/6.1982-1462

Casalino, L., Colasurdo, G., Pastrone, D.: Simple strategy for powered swing-by. J. Guid. Control Dyn. 22(1), 156 (1999a)

Casalino, L., Colasurdo, G., Pastrone, D.: Optimal low-thrust scape trajectories using gravity assist. J. Guid. Control Dyn. 22(5), 637 (1999b)

Clarke, V.C.: A summary of the characteristics of ballistics interplanetary trajectories. Tech. Report no. 32-209, JPL (1962)

Cline, J.K.: Satellite aided capture. Celest. Mech. 19, 405 (1979)

D'Amario, L.A., Byrnes, D.V., Stanford, R.H.: A new method for optimizing multiple-flyby trajectories. J. Guid. Control Dyn. 4, 591 (1981). https://doi.org/10.2514/3.56115

D’Amario, L.A., Byrnes, D.V., Stanford, R.H.: Interplanetary trajectory optimization with application to Galileo. J. Guid. Control Dyn. 5, 465 (1982). https://doi.org/10.2514/3.56194

Deerwester, J.M.: Jupiter swing-by missions to the outer planets. J. Spacecr. Rockets 3(10), 1564 (1966)

Diehl, R., Myers, M.R.: Gravity-assist trajectories to the outer solar system. JPL, D-4677 (1987)

Dunne, J.A., Burgess, E.: The Voyage of Mariner 10. NASA SP, vol. 424 (1978)

Ferreira, A.F.S., Prado, A.F.B.A., Winter, O.C.: A numerical study of powered swing-bys around the Moon. Adv. Space Res. 56(2), 252 (2015). https://doi.org/10.1016/j.asr.2015.04.016

Ferreira, A.F., Prado, A.F.B.A., Winter, O.C., Santos, D.P.S.: Effects of the eccentricity of the primaries in a powered swing-by maneuver. Adv. Space Res. 59(8), 2071 (2017a). https://doi.org/10.1016/j.asr.2017.01.033

Ferreira, A.F., Prado, A.F.B.A., Winter, O.C.: A numerical mapping of energy gains in a powered swing-by maneuver. Nonlinear Dyn. 89(2), 791 (2017b). https://doi.org/10.1007/s11071-017-3485-2

Ferreira, A.F., Prado, A.F.B.A., Winter, O.C.: Planar powered swing-by maneuvers to brake a spacecraft. Comput. Appl. Math. (2017c). https://doi.org/10.1007/s40314-017-0483-4
Flandro, G.: Fast reconnaissance missions to the outer solar system utilizing energy derived from the gravitational field of Jupiter. Acta Astronaut. 12(4), 329 (1966)

Grard, R.: Mercury: the Messenger and BepiColombo missions A concerted approach to the exploration of the planet. Adv. Space Res. 38, 563 (2006). https://doi.org/10.1016/j.asr.2006.06.015

Havnes, O.: The capture of comets by Jupiter. Astrophys. Space Sci. 5(3), 272 (1969)

Hollister, W.M., Prussing, J.E.: Optimum transfer to Mars via Venue. Astron. Acta 12(2), 169 (1966)

Horedt, G.P.: Capture in the restricted three body problem. Acta Astron. 22(1), 55 (1972)

Horedt, G.P.: Numerical exploration of the capture problem. Acta Astron. 24, 207 (1974)

Horedt, G.P.: Capture of planetary satellites. Astron. J. 81(8), 675 (1976)

McNutt, R.L. Jr., Solomon, S.C., Grard, R., Novara, M., Mukai, T.: An international program for mercury exploration: synergy of Messenger and BepiColombo. Adv. Space Res. 33, 2126 (2004). https://doi.org/10.1016/S0273-1177(03)00439-3

McNutt, R.L. Jr., Solomon, S.C., Gold, R.E., Leary, J.C.: The messenger mission to mercury: development history and early mission status. Adv. Space Res. 38, 564 (2006). https://doi.org/ 10.1016/j.asr.2005.05.044

Minovitch, M.A.: A method for determining interplanetary free-fall reconnaissance trajectories. JPL Tec. Memo 312-130, Pasadena (1961)

NASA: LCROSS - Lunar Crater Observation and Sensing SatelliteLCROSS Overview. Page Editor: Robert Garner (2009). Available in: https://www.nasa.gov/mission_pages/LCROSS/ overview/index.html

Negri, R.B., Prado, A.F.B.A., Sukhanov, A.: Studying the errors in the estimation of the variation of energy by the "patched-conics" model in the three-dimensional swing-by. Celest. Mech. Dyn. Astron. (2017). https://doi.org/10.1007/s10569-017-9779-3

Niehoff, J.C.: Gravity-assisted trajectories to solar-system. J. Spacecr. Rockets 3(9), 1351 (1966)

Nock, K.T., Upholf, C.W.: Satellite aided orbit capture. AAS/AIAA paper 79-165 (1979)

Piñeros, J.O.M., Prado, A.F.B.A.: Powered aero-gravity-assist maneuvers considering lift and drag around the Earth. Astrophys. Space Sci. 362(7), 120 (2017)

Prado, A.F.B.A.: Powered swing-by. J. Guid. Control Dyn. 19, 1142 (1996). https://doi.org/10.2514/3.21756

Prado, A.F.B.A.: Close-approach trajectories in the elliptic restricted problem. J. Guid. Control Dyn. 20, 797 (1997). https:// doi.org/10.2514/2.4115

Prado, A.F.B.A.: A comparison of the "patched-conics approach" and the restricted problem for swing-bys. Adv. Space Res. 40, 113 (2007)

Qi, Y., Xu, S.: Mechanical analysis of lunar gravity assist in the EarthMoon system. Astrophys. Space Sci. 360(2), 55 (2015)

Santos, D.P.S., Prado, A.F.B.A., Casalino, L., Colasurdo, G.: Optimal trajectories towards near-Earth-objects using solar electric propulsion (SEP) and gravity assisted maneuver. J. Aerosp. Eng. Sci. Appl. 1(2), 51 (2008)

Silva, A.F., Prado, A.F.B.A., Winter, O.C.: Powered swing-by maneuvers around the Moon. J. Phys. Conf. Ser. 465, 012001 (2013). https://doi.org/10.1088/1742-6596/465/1/012001

Szebehely, V.: Theory of Orbits. Academic Press, New York (1967) 